\section{Information sur le contrôle de qualité dans le laboratoire médical}

QUALAB, Commission suisse pour l'assurance qualité dans le laboratoire médical

La participation aux enquêtes externes est obligatoire pour tous les laboratoires privés, d'hôpitaux et d'officines qui pratiquent des analyses et/ou les facturent à la charge de l'assurance sociale. Cette disposition s'étend aux listes établies par la QUALAB concernant les analyses contrôlées obligatoires. Cette liste ("Contrôle de qualité externe obligatoire») a été publiée dans l'organe de la commission. On peut également la consulter par internet: www.famh.ch/ QUALAB.htm.

Sur mandat de la QUALAB, les diverses organisations de fournisseurs de prestations et les assureurs selon la LAMal, l'AA, l'AM et l'AI, doivent gérer une banque de données établissant la liste de tous les laboratoires et la participation de leurs membres à un contrôle obligatoire de qualité externe. Les laboratoires qui n'y participent pas sont exclus du remboursement par les assureurs, en vertu du concept d'assurance qualité de la QUALAB.

Les organisations de fournisseurs de prestations doivent contrôler cette année la participation, pour l'année 2000, de leurs membres au contrôle de qualité externe. En ce qui concerne les laboratoires de cabinets médicaux, cette tâche a été assumée par le Service tarifaire de la FMH à Oberkirch. Sont avertis tous les exploitants d'un laboratoire de cabinet médical qui ne sont pas annoncés à un centre de contrôle officiel. La saisie des participants n'étant pas toujours sans failles, il est possible que des participants au contrôle de qualité externe aient également été avertis. Les exploitants d'un laboratoire de cabinet médical (cela vaut bien sûr aussi pour ceux qui n'ont pas reçu de lettre sans être toutefois encore inscrits dans un centre de contrôle) ont les possibilités suivantes:

1. Envoi de la confirmation à la participation aux enquêtes externes pour l'année 2000.

2. Inscription dans un centre officiel de contrôle et envoi de la confirmation d'inscription.

3. Envoi, si les analyses sont effectuées dans le laboratoire d'un cabinet de groupe, de la participation avec la désignation de qui est le participant dans ce cabinet de groupe.
4. S'il n'y a pas d'analyses menées et/ou facturées à la charge des assurances sociales, une brève explication par écrit suffit.

5. Une brève explication suffit également si aucune des analyses effectuées est soumise actuellement au contrôle de qualité.

Les détenteurs de laboratoires envoient leurs annonces à l'adresse suivante: Service tarifaire de la FMH, case postale 246, 6208 Oberkirch, tél. 04192500 77, fax 04192105 86, e-mail: fmhtarif@hin.ch.

Si les annonces ne parviennent pas d'ici le 25 juillet 2001 au plus tard, les organisations affiliées doivent annoncer ces laboratoires à la QUALAB qui, de son côté, annonce les laboratoires aux assureurs. Les éventuelles sanctions selon le concept mis au point prendront dès lors effet. Quoiqu'il en soit, les détenteurs de laboratoires qui ne s'annoncent pour les contrôles qu'au milieu de l'an 2001 sont tenus de participer aux autres enquêtes pour 2001. Le contrôle de la participation aux enquêtes 2001 sera répété en 2002.

Le résumé ci-après donne un aperçu sur la conception et le déroulement du contrôle de qualité obligatoire externe.

\section{Contrôle de qualité obligatoire}

\section{Dispositions}

(Extraits du concept QUALAB, non exhaustifs)

- Concept QUALAB, art. 4.2.1.1 al. 1

"Chaque laboratoire participe pour les analyses qu'il effectue à au moins 4 contrôles de qualité externes par an [...].»

- Concept QUALAB, pt 4.2.1.1 al. 7

"Une liste Contrôle de qualité obligatoire fixe les analyses pour lesquelles un contrôle de qualité externe est obligatoire [...].»

- Concept QUALAB, pt 4.2.3.2

"Les laboratoires médicaux qui, en 2 ans, n'ont pas participé aux 8 contrôles prescrits, sont exclus pour une année ou, en cas de récidive, définitivement du remboursement par les assureurs."

- Concept QUALAB, pt 4.2.3.3 al. 1

"Si la précision des mesures par analyse se situe en dehors des critères d'évaluation fixés par la QUALAB (cf. Liste des contrôles de qualité obligatoires), une expertise est ordonnée aux frais du laboratoire médical concerné. L'expertise et les conseils comprennent l'ensemble de l'assurance qualité selon QUALAB et CFLAM."

- Concept QUALAB, pt 4.2.3.3 al. 2

"Le laboratoire médical dont les résultats du contrôle sont insuffisants, est tenu dans tous les cas de chercher aide et conseils." 


\section{Liste des centres de contrôle de qualité reconnus}

Etat au 15 juin 2000

Suisse

a CSCQ (Centre Suisse de Contrôle de Qualité), 2, chemin du Petit-Bel-Air, 1225 Chêne-Bourg, tél. 0223055230 (français), tél. 0223055231 (allemand), tél. 0223055232 (italien), fax 022 30552 38, e-mail: cscq@hcuge.ch, internet: www.cscq.ch b MQ (Verein für medizinische Qualitätskontrolle), Institut für klinische Chemie, Universitätsspital, 8091 Zurich, tél. 0125534 11, fax 0126112 83, e-mail:mq@unizh.ch, internet: www.mqzh.ch

c Zentrallaboratorium BSD SRK AG, Wankdorfstrasse 10, 3000 Berne 22, tél. 03133002 22, fax 0313300633

\section{Etranger}

En suspens.

\section{Liste des analyses soumises au contrôle de qualité externe obligatoire en 2001}

Cette liste entre en vigueur le 1.1.2001.

\begin{tabular}{|c|c|c|c|c|c|}
\hline M & no position & paramètre & $\begin{array}{l}\text { critères } \\
\text { qualité }\end{array}$ & $\begin{array}{l}\text { critères } \\
\text { nombre }\end{array}$ & $\begin{array}{l}\text { CCQ } \\
\text { reconnus }\end{array}$ \\
\hline & 8000.00 & Groupes sanguins & juste & $11 / 12$ & c \\
\hline & 8006.00 & Alanine-aminotransférase (ALAT) & $\pm 25 \%$ & $75 \%$ & $a, b$ \\
\hline $\mathrm{N}$ & 8007.00 & Albumine, chimique & $\pm 15 \%$ & $75 \%$ & $a, b$ \\
\hline \multirow{2}{*}{$\mathrm{N}$} & 8037.00 & Amylase & $\pm 40 \%$ & $75 \%$ & $a, b$ \\
\hline & 8058.00 & Aspartate-aminotransférase (ASAT) & $\pm 25 \%$ & $75 \%$ & $a, b$ \\
\hline $\mathrm{N}$ & 8116.00 & Barbituriques, ql & juste & $75 \%$ & $\mathrm{a}, \mathrm{b}$ \\
\hline \multirow{2}{*}{$\mathrm{N}$} & 8137.00 & Protéine $\mathrm{C}$ réactive (CRP), qn & $\pm 30 \%$ & $75 \%$ & $\mathrm{a}, \mathrm{b}$ \\
\hline & 8137.10 & Protéine $C$ réactive (CRP), par test rapide, sq & $\pm 30 \%$ & $75 \%$ & $a, b$ \\
\hline \multirow[t]{3}{*}{$\mathrm{N}$} & 8149.00 & Calcium total & $\pm 12 \%$ & $75 \%$ & $a, b$ \\
\hline & 8156.00 & Chlorures & $\pm 10 \%$ & $75 \%$ & $a, b$ \\
\hline & 8158.00 & Cholestérol total & $\pm 10 \%$ & $75 \%$ & $a, b$ \\
\hline $\mathrm{N}$ & 8169.00 & Cocaïne, ql & juste & $75 \%$ & $a, b$ \\
\hline \multirow[t]{2}{*}{$\mathrm{N}$} & 8184.00 & Cannabis, ql & juste & $75 \%$ & $a, b$ \\
\hline & 8192.00 & Fer & $\pm 20 \%$ & $75 \%$ & $\mathrm{a}, \mathrm{b}$ \\
\hline \multirow{3}{*}{$\mathrm{N}$} & 8273.00 & Hématocrite & $\pm 12 \%$ & $75 \%$ & $a, b$ \\
\hline & 8275.00 & Hémoglobine & $\pm 12 \%$ & $75 \%$ & $a, b$ \\
\hline & 8284.00 & Urée & $\pm 20 \%$ & $75 \%$ & $a, b$ \\
\hline \multirow[t]{7}{*}{$\mathrm{N}$} & 8288.00 & Cholestérol HDL sans précipitation séparée & $\pm 30 \%$ & $75 \%$ & $\mathrm{a}, \mathrm{b}$ \\
\hline & 8313.00 & lmmunoglobuline IgA (sérum) & $\pm 25 \%$ & $75 \%$ & $a, b$ \\
\hline & 8318.00 & lmmunoglobuline IgG (sérum) & $\pm 25 \%$ & $75 \%$ & $a, b$ \\
\hline & 8321.00 & lmmunoglobuline IgM (sérum) & $\pm 25 \%$ & $75 \%$ & $a, b$ \\
\hline & 8343.00 & Potassium & $\pm 10 \%$ & $75 \%$ & $a, b$ \\
\hline & 8384.00 & Créatine-kinase $(\mathrm{CK})$, total & $\pm 40 \%$ & $75 \%$ & $a, b$ \\
\hline & 8387.00 & Créatinine & $\pm 20 \%$ & $75 \%$ & $a, b$ \\
\hline
\end{tabular}




\begin{tabular}{|c|c|c|c|c|c|}
\hline & 8394.00 & Lactate-déshydrogénase (LDH) & $\pm 25 \%$ & $75 \%$ & $\mathrm{a}, \mathrm{b}$ \\
\hline \multirow[t]{3}{*}{$\mathrm{N}$} & 8403.00 & Leucocytes, répartition & *) & $75 \%$ & $a, b$ \\
\hline & 8406.00 & Leucocytes, numération & $\pm 25 \%$ & $75 \%$ & $\mathrm{a}, \mathrm{b}$ \\
\hline & 8428.00 & Magnésium & $\pm 20 \%$ & $75 \%$ & $\mathrm{a}, \mathrm{b}$ \\
\hline \multirow[t]{2}{*}{$\mathrm{N}$} & 8433.00 & Méthadone, ql & juste & $75 \%$ & $\mathrm{a}, \mathrm{b}$ \\
\hline & 8438.00 & Sodium & $\pm 10 \%$ & $75 \%$ & $\mathrm{a}, \mathrm{b}$ \\
\hline $\mathrm{N}$ & 8444.00 & Opiacés, ql & juste & $75 \%$ & $\mathrm{a}, \mathrm{b}$ \\
\hline \multirow[t]{2}{*}{$\mathrm{N}$} & 8450.00 & Amylase pancréatique & $\pm 25 \%$ & $75 \%$ & $\mathrm{a}, \mathrm{b}$ \\
\hline & 8455.10 & Temps de thromboplastine partielle activée (aPTT) & $\pm 25 \%$ & $75 \%$ & $\mathrm{a}, \mathrm{b}$ \\
\hline \multirow[t]{10}{*}{$\mathrm{N}$} & 8462.00 & Phosphore inorganique & $\pm 15 \%$ & $75 \%$ & $\mathrm{a}, \mathrm{b}$ \\
\hline & 8492.00 & Protéines totales & $\pm 15 \%$ & $75 \%$ & $\mathrm{a}, \mathrm{b}$ \\
\hline & 8548.00 & Quick & $\pm 15 \%$ & $75 \%$ & $\mathrm{a}, \mathrm{b}$ \\
\hline & 8560.00 & Thrombocytes, numération & $\pm 25 \%$ & $75 \%$ & $\mathrm{a}, \mathrm{b}$ \\
\hline & 8572.00 & Triglycérides & $\pm 15 \%$ & $75 \%$ & $\mathrm{a}, \mathrm{b}$ \\
\hline & 8578.00 & Urate & $\pm 20 \%$ & $75 \%$ & $\mathrm{a}, \mathrm{b}$ \\
\hline & 8586.00 & Test de compatibilité type + screen & juste & $100 \%$ & c \\
\hline & 9108.40 & Hépatite B, virus, HBs, recherche des antigènes & pas de faux négatif & $100 \%$ & a, c \\
\hline & 9109.01 & Hépatite C, virus, anticorps Ig ou IgG & pas de faux négatif & $100 \%$ & a, c \\
\hline & 9116.01 & HIV $1+2$, dépistage des anticorps (EIA), ql & pas de faux négatif & $100 \%$ & a, c \\
\hline $\mathrm{N}$ & 9116.40 & HIV $1+2$, dépistage des anticorps (test rapide), ql & pas de faux négatif & $100 \%$ & $\mathrm{a}, \mathrm{b}$ \\
\hline $\mathrm{N}$ & 9309.00 & Urine slide & juste & $75 \%$ & $a, b$ \\
\hline
\end{tabular}

Colonne $\mathrm{M}=$ modifications par rapport à 2000: $\mathrm{N}$ = nouveau sur la liste; $\mathrm{C}=$ changement de critère; $\mathrm{Q}$ = changement de CCQ reconnu. *) critères d'évaluation définis en fonction de la pathologie liée au frottis.

\section{Evaluation du contrôle de qualité obligatoire}

Lors de l'évaluation des résultats du contrôle de qualité externe, il y a lieu de tenir compte des points suivants:

- En médecine de laboratoire, les méthodes et les systèmes analytiques utilisés sont très hétérogènes. Ceci conduit à une hétérogénéité des résultats du contrôle de qualité. Il n'est donc pas possible de réaliser des critères ou des valeurs homogènes.

- La qualité des résultats du contrôle de qualité est influencée par la qualité du matériel de contrôle. Pour des raisons techniques il n'est pas possible de mettre à disposition un matériel de contrôle optimal pour chaque système analytique. Les centres de contrôle de qualité doivent tenir compte de ce facteur lors de l'évaluation des résultats.

- Pour les raisons évoquées ci-dessus, une multitude de cas spéciaux doivent être pris en compte lors de l'évaluation de la qualité des contrôles. Il n'est pas possible de les nommer à l'avance. Pour cette raison et dans le but de permettre des contrôles rapides et administrativement pas trop lourds, une certaine liberté d'appréciation est accordée à titre d'essai pendant un an aux centres de contrôle de qualité. Cette liberté d'appréciation d'un an est également valable pour les paramètres nouvellement introduits. Les centres de contrôle de qualité rapportent à la fin de l'année les cas spéciaux, dans lesquels cette liberté d'appréciation a été utilisée.
4.1 Paramètres avec ou sans contrôle de qualité obligatoire

4.1.1 Paramètres avec contrôle de qualité obligatoire (selon point 3: liste der analyses)

La participation à au moins 4 contrôles est obligatoire. Lorsque la QUALAB n'exige dans des cas exceptionnels pour des analyses spéciales que 2 contrôles, la participation aux 2 contrôles est nécessaire.

La qualité des analyses est évaluée selon les critères de la QUALAB.

4.1.2 Paramètres sans contrôle de qualité obligatoire (ne figurant pas sur la liste du point 3)

Le contrôle de qualité externe est facultatif pour ces paramètres. Il n'existe pas de directives (critères de qualité) de la QUALAB.

\subsection{Critères d'évaluation/certificat}

\subsubsection{Critères de qualité, critères de nombre}

La qualité analytique de chaque paramètre est évaluée au courant de l'année. En règle générale, les conditions sont remplies, lorsque $75 \%$ des résultats des contrôles annuels de chaque paramètre correspondent aux critères de la QUALAB. Certains paramètres peuvent avoir d'autres exigences (cf. colonne "critères de nombre" du tableau à la page 2). 


\subsubsection{Certificat}

Le certificat comprend une liste des paramètres à contrôler (avec numéros de position de la LA), le nombre de contrôles par paramètre, l'évaluation des résultats par contrôle et paramètre (conforme ou non conforme) et une rubrique de texte pour, le cas échéant, l'octroi provisoire du certificat par le centre de contrôle de qualité.

\subsubsection{Nouvelles inscriptions / changement d'inscriptions}

Si un laboratoire introduit une nouvelle analyse ou commence le contrôle de qualité, chaque évaluation fait office de certificat. Les résultats insuffisants doivent être analysés et les mesures prises consignées par le laboratoire.

\subsection{Obligations des laboratoires}

4.3.1 Participation aux contrôles de qualité externes Le laboratoire doit s'inscrire pour toutes les analyses selon le point 4.1.1 ainsi que pour le nombre de contrôles de qualité externes prescrit auprès d'un centre de contrôle de qualité reconnu par la QUALAB. Pour les paramètres de la liste des soins de base (cf. liste fédérale des analyses), seuls des centres de contrôle de qualité suisses sont reconnus. Pour tous les autres paramètres, des centres de contrôle de qualité étrangers peuvent également être reconnus sur demande des sociétés scientifiques.

\subsubsection{Procédure lorsque les critères d'évaluation} ne sont pas remplis

Le laboratoire est tenu de rechercher les causes des résultats insuffisants!

Si les critères d'évaluation pour un paramètre ne sont pas remplis, le laboratoire devrait prendre contact avec le centre de contrôle de qualité pour discuter des problèmes. Le centre de contrôle de qualité confirme par écrit la consultation qui a eu lieu. La consultation fait office de certificat provisoire pour l'année suivante.

$$
\text { Deutsch erschienen in Nr. 31/2001 }
$$

